\title{
Answers to Two Questions Consistently Reflect Cerebral Dysfunction or Damage Subsequent to Mild Closed Head Injuries and Protracted Difficulties with Adaptation
}

\section{Michael A. Persinger}

Clinical Neuroscience Laboratory, Behavioural Neuroscience, Biomolecular Sciences and Human Studies Program Laurentian University, Sudbury, Ontario, Canada

"Corresponding author: Michael A. Persinger, Clinical Neuroscience Laboratory, Behavioural Neuroscience, Biomolecular Sciences and Human Studies Program, Laurentian University, Sudbury, Ontario, Canada, Tel: 01-705-675-4824; Fax: 01-705-671-3844; E-mail: mpersinger@laurentian.ca

Rec date: May 30, 2016; Acc date: Jul 12, 2016; Pub date: Jul 14, 2016

Copyright: (C) 2016 Persinger MA. This is an open-access article distributed under the terms of the Creative Commons Attribution License, which permits unrestricted use, distribution, and reproduction in any medium, provided the original author and source are credited.

\section{Abstract}

The answers to two questions: (1) "Do you feel you are the same person as you were before the cerebral event? And (2) When did the sensed presence begin?" are powerful indicators of cerebral dysfunction during the first two or three years subsequent to significant mechanical impacts to the skull even though traditional neurological screening or examinations are within the normal range. These patients also display protracted difficulties with adaptation and rarely have returned to previous employment. Re-attributing the patient's interpretations of these disturbing experiences from a neurological perspective facilitates adaptation. These interventions might diminish the subjective exacerbations that frequently result in psychiatric referrals years later.

\section{Short Communication}

Because of the capriciousness of mechanical forces and energies distributed through a person's cranium during sudden changes in velocities (such as motor vehicle incidents) the range of long-term disability is sometimes difficult to predict with precision. A significant proportion of patients whose GCS ratings were $>13$ within a few hours after the cerebral impact and who exhibited all of the signs of a potentially excellent recovery subsequently exhibit protracted incapacities, loss of employment, and delayed psychiatric issues. Between the first neurological examination following the event and the psychiatric variants that may have occurred after several years, thorough neurology-based neuropsychological examinations revealed substantial impairment [1]. Recent precision of QEEG (Quantitative Electroencephalography) and LORETA (Low Resolution Electromagnetic Tomography) to reveal increment shifts in frequencydependent current density within the cerebral volume have verified this approach [2].

I carefully reviewed the cases files (from the last 30 years of practice) of about 800 of our patients who were between the ages of 18 and 80 years at the time of a full neuropsychological (2 days) assessment. They had sustained "mild to moderate" closed head injuries with or without suspensions of consciousness. Many of these patients still exhibited minimal "recovery", that is, failure to return to previous perceptions of capability and employment. Two questions have discriminated with more than $95 \%$ accuracy whether or not the patients displayed neuropsychological impairment even though neurological screenings were within normal or age-appropriate limits. These two questions were: 1) Are you the same person now as you were before the incident? and 2) When did the sensed presences begin?

If the person says no the first and gives specifics to the second, the likelihood of a significant neuropsychological impairment was greater than 95\%. These patients reported a sensed presence that was perceived to occur along the left side of the body in the peripheral visual field. It usually occurred after a day of travelling and often at night between 02 and $04 \mathrm{hr}$ when the patient "suddenly awoke". The gender of the "presence" was primarily the opposite of that of the patient. The cause was attributed to culturally-congruent sources such as an angel, a deceased member of the family, or ancestor-tribal member. This pattern of responding was also associated with unusual elevations of complex partial epileptic-like indicators and scaled psychiatric indicators (for example the Minnesota Multiphasic Personality Inventory, MMPI) that remain abnormal for years. There were also profound changes in personality that were strongly coupled to the approximately $70-80 \%$ dissolution of previous relationships (marriages, common law arrangements) within two to four years after the incident even though this "bonding" had been previously stable for years to decades [3].

One example was an 18 year old woman who had sustained right parietotemporal injury (with transient contralateral hemiparesis) during a motor vehicle incident. About one month after taken the incident she began to suddenly awaken between 02 and $04 \mathrm{hrs}$ and experience intense fear and a male presence that was localized to her left side. The parents and the patient attributed the experience to a dead relative and felt the patient would soon be "taken. For the majority of these patients a left-sided sensed presence was associated with apprehension; a right-sided sensed presence was more likely to be associated with positive experiences and the patient's name being "called" during twilight states. All of these patients "wanted their old self back" and reported symptoms consistent with the classic grieving sequence of maintained behavior ("denial"), irritability, depression, and acceptance except it appeared to be the "loss of the sense of self" that was being grieved. The patients appeared maintained within the third stage.

I have found that the vast majority of the patients had never revealed the occurrence of the sensed presences to any of the many medical professionals they had seen even though the experiences were often terrifying and contributed to the patient's distress [4]. The patients were concerned they would be labelled as "crazy". A very significant sequence is to have the patient acknowledge that the sense 
Citation: Persinger MA (2016) Answers to Two Questions Consistently Reflect Cerebral Dysfunction or Damage Subsequent to Mild Closed Head Injuries and Protracted Difficulties with Adaptation. J Neurol Disord 4: 277. doi:10.4172/2329-6895.1000277

Page 2 of 2

of self and all experiences are determined by cerebral functions. I usually ask the patient to point to "Bill" or "Alice" (whatever the first name might be). Usually the person points to the chest region. I emphasize that any of the person's organs can be replaced and he or she would be the same person. What cannot be replaced is the brain because the brain is the person.

Once they appreciate that the functions strongly associated with the left hemisphere in most people are associated with the experiences of the self and awareness and experiences classically associated with the right hemisphere in most people are associated with the sensed presence and other intrusions the apprehension is minimized. The effect is enhanced when simple predictions are given with respect to when and where these experiences have occurred. The rational neurological attributions facilitate much greater adaptation than belief, cultural traditions, or idiosyncratic explanations. We have development a specific technology that can elicit the sensed presence in the clinical setting so that alternative attributions by the patient can be acquired and the apprehension can be reduced. This experimental induction has been accomplished by applying physiologically patterned weak magnetic fields across the temporal plane [5]. Despite the brevity of the intervention, it is very effective [6].

\section{References}

1. Persinger MA (1995) Clinical neurological indicators are only moderately correlated with quantitative neuropsychological test scores in patients who display mild-moderate brain impairment following closed head injuries. Percep Mot Skil 81: 1283-1292.

2. Corradini PL, Persinger MA (2015) Replace psychometric inferences with direct brain measurements: LORETA reflects traditional cerebral loci for neuropsychological tests. Neurosci Med 6: 107-115.

3. Tiller S, St-Pierre LS, Persinger MA (2013) Absence of quantitative improvement in neuropsychological profiles in patients who exhibit moderate brain impairment: comparisons of cross-sectional and longitudinal data (1 to 4 years post injury). J Behav Brain Sci 3: 225-228.

4. Persinger MA (2001) The neuropsychiatry of paranormal experiences. J Neuropsychiat Clin Neurosci 13: 515-524.

5. Persinger MA, Healy F (2002) Experimental facilitation of the sensed presence: possible intercalation between hemispheres induced by complex magnetic fields. J Ner Men Dis 190: 533-541.

6. Persinger MA (1993) Personality changes following brain injury as a grief response to the loss of sense of self: phenomenological themes as indices of local lability and neurocognitive structuring as psychotherapy. 